Article

\title{
Broadband Impedance Measurement of Lithium-Ion Battery in the Presence of Nonlinear Distortions
}

\author{
Jussi Sihvo $^{1, *(\mathbb{D} \text {, Tomi Roinila }}{ }^{1}(\mathbb{D})$ and Daniel-Ioan Stroe ${ }^{2}(\mathbb{D}$ \\ 1 Department of Electrical Engineering, Tampere University, Korkeakoulunkatu 7, 33720 Tampere, Finland; \\ tomi.roinila@tuni.fi \\ 2 Department of Energy Technology, Aalborg University, Pontoppidanstræde 101, 9220 Aalborg, Denmark; \\ dis@et.aau.dk \\ * Correspondence: jussi.sihvo@tuni.fi
}

Received: 16 April 2020; Accepted: 12 May 2020; Published: 15 May 2020

\begin{abstract}
The impedance of a Lithium-ion (Li-ion) battery has been shown to be a valuable tool in evaluating the battery characteristics such as the state-of-charge (SOC) and state-of-health $(\mathrm{SOH})$. Recent studies have shown impedance-measurement methods based on broadband pseudo-random sequences (PRS) and Fourier techniques. The methods can be efficiently applied in real-time applications where the conventional electrochemical-impedance spectroscopy (EIS) is not well suited to measure the impedance. The techniques based on the PRS are, however, strongly affected by the battery nonlinearities. This paper presents the use of a direct-synthesis ternary (DST) signal to minimize the effect caused by the nonlinearities. In such a signal, the second- and third-order harmonics are suppressed from the signal energy spectrum. As a result, the effect of the secondand third-order nonlinearities are suppressed from the impedance measurements. The impedance measurements are carried out for a nickel manganese cobalt Li-ion battery cell. The performance of the method is compared to the conventional EIS, as well as to other PRS signals which are more prone to battery nonlinearities. The Kronig-Kramers (K-K) transformation test is used to validate the uniqueness of the measured impedance spectra. It is shown that the measurement method based on the DST produces highly accurate impedance measurements under nonlinear distortions of the battery. The method shows a good $\mathrm{K}-\mathrm{K}$ test behavior indicating that the measured impedance complies well to a linearized equivalent circuit model that can be used for the SOC and $\mathrm{SOH}$ estimation of the battery. Due to the good performance, low measurement time, and simplicity of the DST, the method is well suited for practical battery applications.
\end{abstract}

Keywords: Li-ion batteries testing; impedance measurements; pseudo random sequences

\section{Introduction}

Lithium-ion (Li-ion) batteries have become increasingly important in various portable and stationary electrical energy applications in recent years [1]. This is due to their high energy and power density and constantly reducing prices. However, constant monitoring of the battery state is required in order to guarantee their safe and stable operation in the application. The battery state is typically characterized in terms of state parameters, such as state-of-charge (SOC) and state-of-health (SOH), which are typically estimated from battery voltage, current, and temperature measurements [2,3].

In recent years, the battery AC impedance has been shown to provide valuable information about the SOC and SOH of the battery [3,4]. The battery impedance is typically obtained by the electrochemical-impedance-spectroscopy (EIS), which utilizes a sinusoidal single-frequency perturbation injected in the battery [5-7]. The method is very accurate and reliable due to its high 
signal-to-noise-ratio (SNR). However, the implementation of the EIS for practical battery applications is difficult due to the long measurement time and the complexity of the sinusoid generation [3]. To overcome the limitations of the conventional EIS, broadband methods such as multisines and pseudo-random-sequences (PRS) have been studied for battery impedance measurements [8-11]. From these methods, especially the PRS are able to overcome the drawbacks of the EIS as the PRS methods are fast, relatively accurate, and easy to implement to a real application due to only a low number of required perturbation signal levels.

The Li-ion batteries are highly time-variant and nonlinear systems which affect the accuracy of the impedance measurements [5,12]. While the EIS is relatively immune to battery nonlinearities, broadband methods, such as PRS methods, are often significantly affected by the presence of the nonlinearities. To mitigate the effect of the nonlinear distortion, some PRS signals can be designed to have zero energy at specific harmonic components which prevents nonlinear distortion in these harmonics [13-18]. The most dominating nonlinearities are usually related to even-order harmonics, and suppression of these harmonics has been shown to improve the performance of the battery impedance measurements [10]. Studies have shown that suppressing the third-order harmonics in addition to the second-order harmonics will increase the measurement accuracy even more in some nonlinear systems [16,17]. An example of a PRS signal with supressed harmonic multiples of two and three is the direct-synthesis-ternary (DST) sequence [17]. Due to the good nonlinear system capabilities and other broadband characteristics, the DST sequence is a potential alternative for impedance measurements in practical battery applications.

This paper demonstrates the use of the DST sequence with suppressed harmonic multiples of two and three for impedance measurements of a nickel-manganese-cobalt Li-ion battery. The performance of the DST sequence is compared to the conventional EIS, as well as to two other PRS sequences: the maximum-length-binary (MLB) and the quadratic-residue-ternary (QRT) sequences, which are more prone to battery nonlinearities. The Kronig-Kramers (K-K) transformation test is used to validate the uniqueness of the measured impedance spectra $[19,20]$. It is shown that the DST sequence method provides the impedance measurements with the least deviation and lowest error compared to the other PRS methods. In addition, it is shown that the accuracy of the PRS methods can be increased by measuring the frequency band of interest adjacently in smaller sub-bands.

The rest of the paper is organized as follows. Section 2 discusses the impedance measurement of a battery under nonlinear distortions. The generation and design of the utilized PRS signals is carried out in Sections 3 and 4, respectively. The experimental arrangement is presented in Section 5. The results are presented and discussed in Section 6 and the conclusions are drawn in Section 7.

\section{Nonlinear System Measurements}

A typical nonlinear system can be represented as in Figure 1 [16]. When applying a perturbation signal $u(t)$ to the system input, a response signal $y(t)$ is created in the system output according to the impulse response $h(t)$ of the system. By applying a Fourier transform to the system input and output signal measurements, the impulse response can be obtained in the frequency domain according to Equation (1) [13]. For nonlinear systems, the impulse response includes the system linear part along with the even- and odd-order nonlinear distortion dynamics. In addition, the system input and output signals are also affected by noise which distorts the uniformity of the measured impulse response:

$$
H(j \omega)=\frac{Y(j \omega)}{U(j \omega)}
$$

The system arrangement in Figure 1 can also be applied to battery impedance measurements as batteries are regarded highly nonlinear systems [5]. The battery impedance can be treated as the impulse response which can be obtained by using the current as the perturbation signal and measuring the voltage. The conventional method for battery impedance measurements is 
the EIS which utilizes a single sine perturbation [5-7,12]. The method produces accurate results, and is relatively resilient to the nonlinear distortions because the signal energy is concentrated on the fundamental component of the excited frequency spectrum [13]. Therefore, other harmonics have very low energy preventing the nonlinear distortion from affecting the fundamental component.

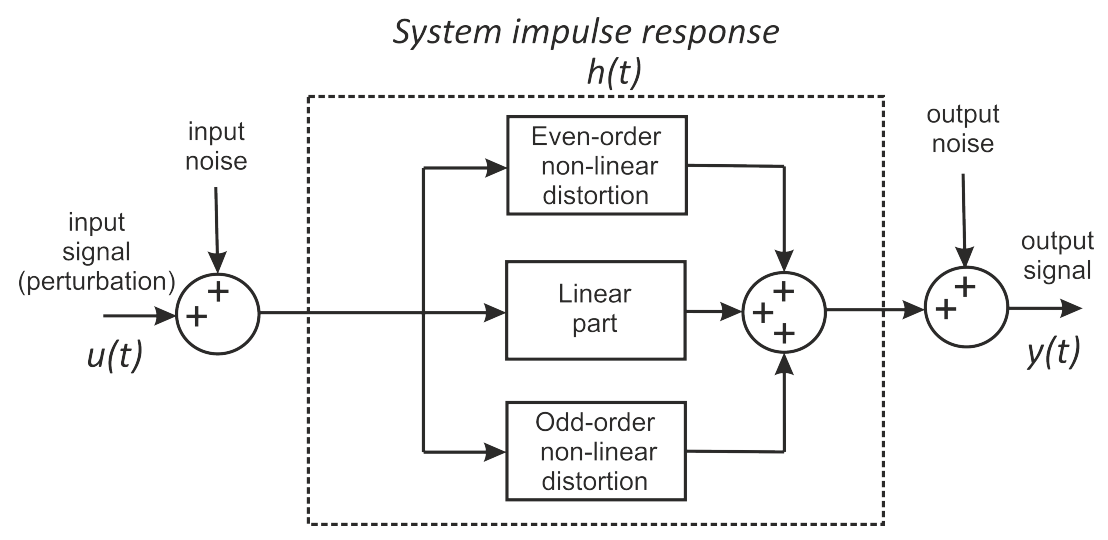

Figure 1. Typical nonlinear system arrangement.

Despite the good performance of the EIS, the method is not well suited for practical applications due to the complexity of the sinusoid generation [3]. Moreover, the method is very slow as each frequency harmonic must be separately excited. To overcome the foregoing limitations of the EIS for practical applications, the broadband PRS signals can be used due to their low complexity and low measurement time [9-11]. The PRS signals, however, are usually prone to the system nonlinearities as they have energy at several harmonics $[13,15]$. This increases the dynamics of the even- and odd-order nonlinear distortions, which, along with the linear part, are mixed in the measurements [16].

The performance of the PRS signals for nonlinear system identification can be increased by suppressing the energy of the specific harmonic components of the perturbation to zero [15-17]. In particular, the suppression can be applied to harmonic multiples of two which covers all the components affecting the even-order nonlinear distortion $[15,16,18]$. With zero energy at these components, the even-order nonlinear distortion is not present in the measured impedance spectra. This type of PRS signal has been recently used for battery impedance measurements where it has shown good performance [10]. The effect of nonlinear distortions can be further reduced by suppressing the third-order harmonics in addition to the second-order harmonics. One such a perturbation is known as a direct-synthesis-ternary (DST) signal that is shown to provide accurate measurements in some nonlinear system case studies [16,17].

\section{Generation of the Used PRS Signals}

The PRS signals are broadband signals which have uniformly distributed energy spectra and finite number of signal levels (usually two or three levels). Therefore, these signals provide fast and relatively accurate measurements and can be injected into a system using low-cost signal generators. Thus, the PRS signals are attractive for practical implementation in various applications, including batteries. In this section, a generation of three different PRS with different harmonic multiples suppressed are introduced. The sequences are chosen to have the following harmonic suppression: sequence without any suppression, sequence with harmonic multiples of two suppressed, and sequence with harmonic multiples of two and three suppressed.

In order to achieve the suppression of harmonic multiples of two for the PRS, the signal Fourier transform $U_{\text {PRS }}(k)$ must have the frequency domain properties as in (2). In order to satisfy 
the conditions in (2), the time domain sequence $u_{\mathrm{PRS}}(t)$ must have inverse-repetitive properties given in (3) $[14,16]$ :

$$
\begin{gathered}
\left|U_{\mathrm{PRS}}(k)\right|=0 \quad \text { for } k=2,4,6,8,10 \ldots \\
u_{\mathrm{PRS}}(i)+u_{\mathrm{PRS}}(i+N / 2)=0
\end{gathered}
$$

For the PRS having harmonic multiples of two and three suppressed, along with (2) and (3), the sequence must have additional properties in the time and frequency domains given in (4) and (5). It should be noted that the sequence must have at least three signal levels in order to satisfy $(5)[14,17]$ :

$$
\begin{gathered}
\left|U_{\mathrm{PRS}}(k)\right|=0 \quad \text { for } k=3,6,9,12,15 \ldots \\
u_{\mathrm{PRS}}(i)+u_{\mathrm{PRS}}(i+N / 3)+u_{\mathrm{PRS}}(i+2 N / 3)=0
\end{gathered}
$$

For the selection of the PRS signals satisfying the above criteria, various types of PRS generation algorithms have been introduced [13-17]. In this paper, the direct-synthesis-ternary (DST) sequence is used as the signal to meet the conditions in (2)-(5). Therefore, it has the best properties for the mitigation of the system nonlinear distortion. The quadratic-residue-ternary (QRT) sequence is used as the signal to meet the conditions in (2) and (3) for the suppression of harmonic multiples of two. Thus, the signal has some properties for the mitigation of the system nonlinear distortion. Finally, the maximum-length-binary (MLB) sequence is used as the signal without harmonic suppression. Therefore, the MLB sequence has the weakest nonlinear system measurement properties. The properties and generation algorithms of the MLB, QRT and DST signals are presented in the following sub-sections.

\subsection{Maximum-Length-Binary (MLB) Sequence}

The MLB sequence theory is based on the linear recurrence relation algorithm defined as in (6) [13]. The first $n$ elements in $u_{\mathrm{MLB}}$ must be initialized to any combination of 0 and 1 excluding the zero vector. The $C$ contains the coefficients of the primitive polynomial of order $n$ which can be freely selected by the user. The length of the MLB sequence is determined as $N=2^{n}-1$, thus having a very limited amount of possible $N$. As a result, the algorithm creates a binary sequence of values 0 and 1 which are usually mapped to -1 and 1 . The sequence harmonic components has uniform magnitudes determined by the sequence amplitude $A$ and $N$ given in (7) [13,14]:

$$
\begin{gathered}
u_{\mathrm{MLB}}(i)=\sum_{r=1}^{n} C_{r} u_{\mathrm{MLB}}(i-r), \quad \bmod 2, \\
\left|U_{\mathrm{MLB}}(k)\right|=\sqrt{N+1} A
\end{gathered}
$$

\subsection{Quadratic-Residue-Ternary (QRT) Sequence}

The QRT sequence can be created according to the quadratic residue code [16]. The code creates a repetitive sub-sequence $v(i), i=1,2 \ldots N / 2$, where $N / 2$ is prime, as follows:

$$
\begin{array}{lll}
v(i)=1 & \text { if } i \in\left\{1^{2}, 2^{2}, 3^{2}, \ldots,(N / 2)^{2}\right\} & \bmod N / 2 \\
v(i)=-1 & \text { if } i \notin\left\{1^{2}, 2^{2}, 3^{2}, \ldots,(N / 2)^{2}\right\} & \bmod N / 2 \\
v(i)=0 & \text { if } i=N / 2 &
\end{array}
$$


The sub-sequence $v(i)$ is then concatenated two times to form a sequence $s(i)=\left[\begin{array}{ll}v(i)_{(1)} & v(i)_{(2)}\end{array}\right]$ from which the QRT-sequence can be obtained by inverting the alternate elements in $s(i)$ as

$$
u_{\mathrm{QRT}}(i)=-1^{i-1} s(i) \quad i=1,2 \ldots N .
$$

As a result, the generated QRT-sequence $u_{\mathrm{QRT}}$ has three signal levels and zero power at all even-order harmonics. The only limitation for the possible $N$ values is that the $N / 2$ must be a prime which gives freedom to select suitable $N$ for an application. The non-zero harmonic of the QRT sequence has magnitudes determined by the sequence amplitude $A$ and $N$ as given in (9) [14-16]:

$$
\left|U_{\mathrm{QRT}}(k)\right|=\sqrt{2 N} A \quad \text { for } k=1,3,5,7 \ldots
$$

\subsection{Direct-Synthesis-Ternary (DST) Sequence}

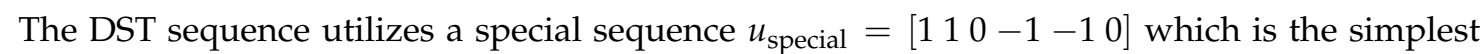
possible sequence with harmonic multiples of two and three suppressed. In addition, a basic sequence is required for the generation. Suitable basic sequences are introduced in [17], one of which is the sub-sequence $v(i)$ used for QRT sequence in Section 3.2 which is also utilized here. The basic sequence $v(i)$ of length $N_{\mathrm{v}}$ is concatenated six times to create a sequence $a=\left[\begin{array}{lllll}v_{(1)}(i) & v_{(2)}(i) & \ldots & v_{(6)}(i)\end{array}\right]$. The special sequence is concatenated $N_{\mathrm{v}}$ times to create a sequence $b=\left[\begin{array}{llll}u_{\text {special }(1)} & u_{\text {special }(2)} & \cdots & u_{\text {special }\left(N_{\mathrm{v}}\right)}\end{array}\right]$. With equal lengths of the sequences $a$ and $b$, the DST sequence can then be formed according to (10) [17].

$$
u_{\mathrm{DST}}(i)=a(i) * b(i)
$$

As a result, the generated DST-sequence $u_{\text {DST }}$ has three signal levels and zero power at all harmonic multiples of two and three. Its non-zero harmonic components have magnitude spectrum values determined by the sequence amplitude $A$ and sequence length $N$ given in (11) [17]:

$$
\left|U_{\mathrm{DST}}(k)\right|=\sqrt{2 N} A \quad \text { for } k=1,5,7,11 \ldots
$$

\section{Perturbation Design}

For the PRSs, the length of the sequence $N$ and the generating frequency $\left(f_{\text {gen }}\right)$ define the lowest frequency harmonic of the sequences as in (12). The power spectrum of the sequences are determined by (13) [14]:

$$
\begin{gathered}
f_{\min }=\frac{f_{\text {gen }}}{N} . \\
P_{\mathrm{PRS}}(k)=2\left(\frac{\sin (\pi k / N)}{\pi k / N} \frac{\left|U_{\mathrm{PRS}}(k)\right|}{N}\right)^{2} \text { for } 1,2,3 \ldots, N
\end{gathered}
$$

Figure 2 shows an example of the MLB, QRT, and DST both in the time domain and frequency domain. The signal lengths are chosen to be approximately the same ( 63 for the MLB, 62 for the QRT, and 66 for the DST). All signals are generated at $60 \mathrm{~Hz}$. As the figure shows, all the even-order harmonics are suppressed in the QRT, and all the even- and third-order harmonics are suppressed in the DST. The figure also shows that the power of the QRT and DST sequences are similar due to the fact that the magnitudes of the harmonics in (9) and (11) are the same. However, small reduction in the power for the DST sequence can be observed, which is due to the slightly longer $N$. As a drawback of the harmonic suppression, the frequency resolution of the QRT and DST sequence measurements becomes more sparse as the zero-harmonics must be removed from the measured spectra. Despite the consistent shape of the non-zero harmonics for all PRSs, the magnitudes will 
eventually decrease to zero at $f_{\text {gen }}$. This limits the highest frequency of the measurements as given in (14) [13]:

$$
f_{\max }=0.45 f_{\text {gen }}
$$

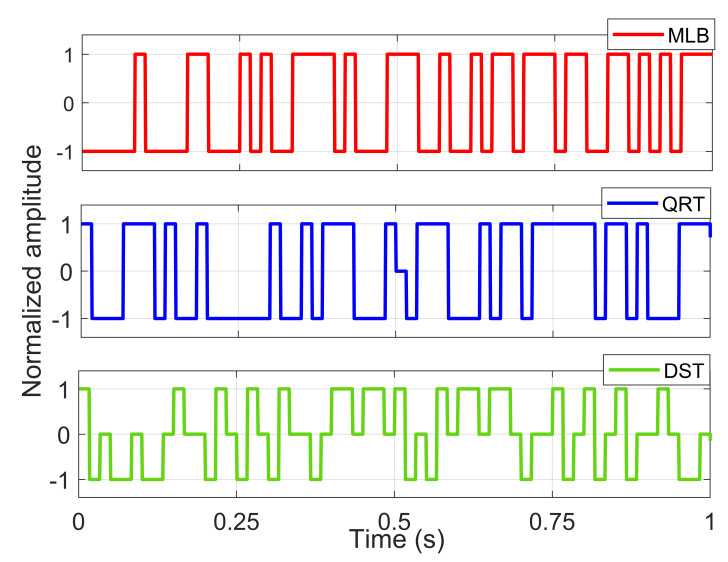

(a)

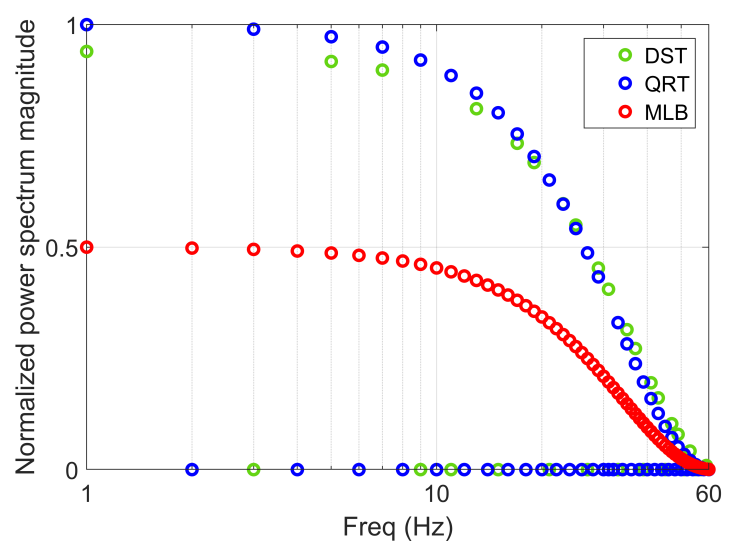

(b)

Figure 2. Sample of MLB, QRT, and DST sequences in the time domain and in the frequency domain $\left(f_{\text {gen }}=60 \mathrm{~Hz}\right)$. (a) Time domain; (b) Frequency domain.

The desired bandwidth of the measurements $\left(f_{\min }-f_{\max }\right)$ is determined by (12) and (14) from which suitable $N$ and $f_{\text {gen }}$ can be solved. For battery impedance measurements, the bandwidth of interest depends highly on the battery chemistry and what regions of the impedance are desired to be captured $[5,10]$. In the case of the NMC battery cell used in this study, the bandwidth of interest was empirically found to be approximately between $200 \mathrm{mHz}$ and $2.5 \mathrm{kHz}$.

For practical implementation, the sequences should have high SNR, which is determined by the sequence amplitude $A$ and length $N$. The amplitude of the sequence is usually restricted by the application where the measurements are implemented. Moreover, the system nonlinear effects are increased with too high amplitudes, which is also the case for battery measurement [21]. Therefore, $N$ offers a possibility to increase the SNR without adjusting the amplitude. The sequence power in (13) can be increased when reducing $N$ which also increases the SNR as it is directly proportional to the signal power [10]. To implement this in practice, the bandwidth of the measurements can be divided into sub-bands each of which is measured consecutively with shorter $N$. The obtained sub-bands can then be united to obtain the desired bandwidth. Apart from the SNR increase, the frequency resolution can this way be set more conveniently for each sub-band. This significantly reduces the amount of measurement data compared to measurements carried out with one long sequence to cover the whole bandwidth. The measurement time is also slightly increased but not drastically as it is still dictated by the time constant of the lowest frequency harmonic of the bandwidth.

\section{Experiments}

In the experiments, the impedance of a nickel-manganese-cobalt (NMC) Li-ion battery cell with a nominal voltage of $3.7 \mathrm{~V}$ and a capacity of $2.7 \mathrm{Ah}$ was measured at $25^{\circ} \mathrm{C}$. The measurements were carried out at various SOCs by three different PRS signals: the MLB, the QRT, and the DST, along with the conventional EIS used as a reference. The scheme of the experimental setup is shown in Figure 3 where PRS signals and the EIS were injected to the battery as a current reference for the bi-directional power supply by using a data acquisition device. The offset of the current perturbation is set to zero in order to assure that the $\mathrm{SOC}$ is not changing during the measurements. The measurements were carried out in the SOC range $20-80 \%$, with $20 \%$ SOC resolution. The transition between the SOCs was performed by discharging the cell to the desired SOC with a current of $1 \mathrm{C}$. Before the measurements, 
a relaxation time of one minute was applied to the cell. It is suggested that the relaxation time should be several hours to guarantee the equilibrium state of the battery cell, but these long relaxation times are not feasible in practical application [6]. Therefore, the relaxation time was kept the same in order to get consistent results despite the non-equilibrium state of the battery cell. During the time of the current perturbation, the battery current and voltage were measured and the data acquisition device was used to gather the data for impedance calculations and frequency response analysis.

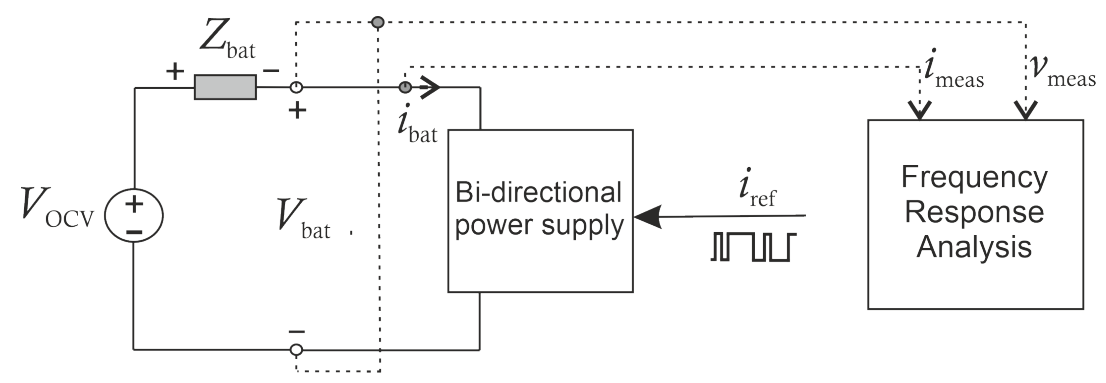

Figure 3. Experimental setup scheme.

The PRS measurements were carried out by using two different configurations. First, the measurements were carried out with a long sequence covering the designed bandwidth. This configuration is regarded as full-band measurements in further parts of this paper. Second, the bandwidth of the measurements was divided into two sub-bands measured adjacently by using a shorter sequence with different generation frequencies. The sub-bands were then united to cover the designed bandwidth. This configuration is regarded as sub-band measurements in further parts of this paper. The bandwidths of the full-band and sub-band measurements along with the design parameters of the PRS signals and the EIS are presented in Table 1. For the PRS measurements, no averaging or filtering was applied. For the EIS, 40 adjacent periods were used for averaging in order to provide smoothed and reliable reference for the PRS measurements. The PRS signals were generated by using the program available in [15] with the extension to the DST generation from [17].

Table 1. Design parameters for the measurements

\begin{tabular}{|c|c|c|c|c|}
\hline Sequence & Amplitude & $f_{\text {gen }}$ & $N$ & $f_{\min }-f_{\max }$ \\
\hline \multicolumn{5}{|c|}{ Full-band parameters } \\
\hline MLBS & $2 \mathrm{~A}$ & $6 \mathrm{kHz}$ & 32767 & $180 \mathrm{mHz}-2.7 \mathrm{kHz}$ \\
\hline QRT & $2 \mathrm{~A}$ & $6 \mathrm{kHz}$ & 32762 & $180 \mathrm{mHz}-2.7 \mathrm{kHz}$ \\
\hline DST & $2 \mathrm{~A}$ & $6 \mathrm{kHz}$ & 32826 & $180 \mathrm{mHz}-2.7 \mathrm{kHz}$ \\
\hline EIS & $2 \mathrm{~A}$ & - & - & $150 \mathrm{mHz}-2.7 \mathrm{kHz}$ \\
\hline \multicolumn{5}{|c|}{ low-frequency sub-band parameters } \\
\hline MLBS & $2 \mathrm{~A}$ & $100 \mathrm{~Hz}$ & 2047 & $49 \mathrm{mHz}-45 \mathrm{~Hz}$ \\
\hline QRT & $2 \mathrm{~A}$ & $100 \mathrm{~Hz}$ & 2042 & $49 \mathrm{mHz}-45 \mathrm{~Hz}$ \\
\hline DST & $2 \mathrm{~A}$ & $100 \mathrm{~Hz}$ & 2082 & $48 \mathrm{mHz}-45 \mathrm{~Hz}$ \\
\hline \multicolumn{5}{|c|}{ high-frequency sub-band parameters } \\
\hline MLBS & $2 \mathrm{~A}$ & $10 \mathrm{kHz}$ & 2047 & $4.9 \mathrm{~Hz}-4.5 \mathrm{kHz}$ \\
\hline QRT & $2 \mathrm{~A}$ & $10 \mathrm{kHz}$ & 2042 & $4.9 \mathrm{~Hz}-4.5 \mathrm{kHz}$ \\
\hline DST & $2 \mathrm{~A}$ & $10 \mathrm{kHz}$ & 2082 & $4.8 \mathrm{~Hz}-4.5 \mathrm{kHz}$ \\
\hline
\end{tabular}

The error analysis of the measurements is carried out in terms of normalized-root-mean-square-error (NRMSE) given in (15) where $Z_{\mathrm{PRS}}$ is the impedance from PRS measurements, $Z_{\mathrm{EIS}}$ is the impedance from EIS measurements, and $l$ is the length of the impedance vectors. The maximum absolute difference in $Z_{\mathrm{EIS}}$ is used as a normalization factor in order to present the errors in percentages:

$$
\mathrm{NRMSE}=\frac{\sqrt{\frac{1}{l} \sum_{i=1}^{l}\left(\left\|Z_{\mathrm{PRS}}(i)\right\|-\left\|Z_{\mathrm{EIS}}(i)\right\|\right)^{2}}}{\max \left(\left\|Z_{\mathrm{EIS}}\right\|\right)-\min \left(\left\|Z_{\mathrm{EIS}}\right\|\right)}
$$


To analyze the uniqueness and goodness of the measured impedances, the Kronig-Kramers compliance test was applied to the measured data. The Kronig-Kramers test utilizes the theory which states that the imaginary part of the impedance data point can be estimated according to the corresponding real part and vice versa $[19,20]$. In the method, each data point is fitted to a linear equivalent circuit model. The real and imaginary part residual errors from the test, given in (16), should then be relatively small and randomly distributed along the frequency axis to guarantee the data are $\mathrm{K}-\mathrm{K}$ compliant. On the contrary, systematic residual errors indicate that the data are corrupted. Therefore, the test is considered to provide good indication of the uniqueness of the PRS measurements and the effect of nonlinear distortion. The test is applied by using a software available in [19] where the number of K-K elements is optimized adaptively to avoid both under and overfitting of the $\mathrm{K}-\mathrm{K}$ transforms:

$$
\Delta_{\mathrm{re}}(j \omega)=\frac{Z_{\text {meas-re }}(j \omega)-Z_{\mathrm{kk}-\mathrm{re}}(j \omega)}{\left|Z_{\mathrm{kk}}(j \omega)\right|}, \quad \Delta_{\mathrm{im}}(j \omega)=\frac{Z_{\text {meas-im }}(j \omega)-Z_{\mathrm{kk}-\mathrm{im}}(j \omega)}{\left|Z_{\mathrm{kk}}(j \omega)\right|} .
$$

\section{Results and Discussion}

Figure 4 shows full-band battery impedance measurements at different SOCs obtained by the PRS perturbations and the EIS. All the impedance results obtained with PRS techniques are similar to the results obtained using the traditional EIS technique. However, the increased performance of the DST sequence over other PRS can be concluded as it has the best match to the EIS results and it has the smallest deviation compared to the MLB and QRT sequences. The deviation of the QRT sequence at low frequencies (right-hand side of the plot) seems to be systematic indicating the presence of the odd-order nonlinearities. This cannot be explained by the SNR as it is the same for DST and QRT. The MLB sequence produces the least accurate impedance measurements compared to the EIS. Therefore, it can be concluded that the battery cell under study exhibits relatively strong nonlinear distortions affecting the measurements.

The sub-band measurements at different SOC are shown in Figure 5. The sub-band measurements have no significant effect on the performance of the DST, which still provides accurate results. However, it can be seen that the deviation of QRT and MLB at the low frequencies (semicircle) is reduced compared to the full-band measurements. This is expected to be caused by the increased SNR, which is approximately 16-times greater compared to the full-band measurements. In particular, the performance of the MLB is significantly increased as the impedance obtained by the MLB sequence now more accurately follows the results obtained by the EIS and DST. Therefore, it can be concluded that the nonlinear distortion effects can be somewhat reduced by using sub-band measurements.

Figure 6 shows the bode representation of the impedances of both full-band and sub-band measurements which correspond to the complex-plane representation of Figures 4 and 5 . The magnitude spectrums at $40 \%, 60 \%$, and $80 \%$ of SOC underlines the accurate performance of the DST as the curve very accurately follows the corresponding curve obtained by the EIS. For measurements at $20 \%$ of SOC, the magnitude responses of the PRS measurements are differing from the EIS, which states that the measurement conditions might not have been the same for the PRSs and the EIS. The phase response reveals that all the PRSs have very small offset with respect to the phase response of the EIS at frequencies below $50 \mathrm{~Hz}$. This explains why the PRS measurements are not exactly following the EIS measurements in the complex-plane even at other than $20 \%$ of SOC. This is most likely caused by either small differences in the measurement conditions between the PRS signals and the EIS, or by the nonlinear distortion still being present even for the DST sequence. Between $50 \mathrm{~Hz}$ and $1 \mathrm{kHz}$, the obtained results using the PRS techniques accurately follow the EIS results. At frequencies above $1 \mathrm{kHz}$, the phase difference is increasing, which is most likely caused by the bi-directional power supply inability to amplify the injected perturbations correctly for these frequencies. For the sub-band measurements, the impedance magnitudes obtained by the MLB and the QRT are more accurately following the results obtained by the EIS as was the case in the complex-plane impedances. Still, it can be concluded that especially the DST captures 
the shape of the magnitude and phase response at the same frequency harmonics as the EIS, which also indicates a good performance of the method.

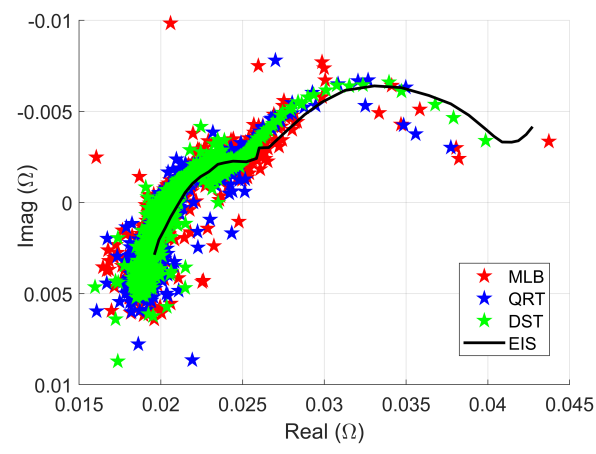

(a) SOC $20 \%$

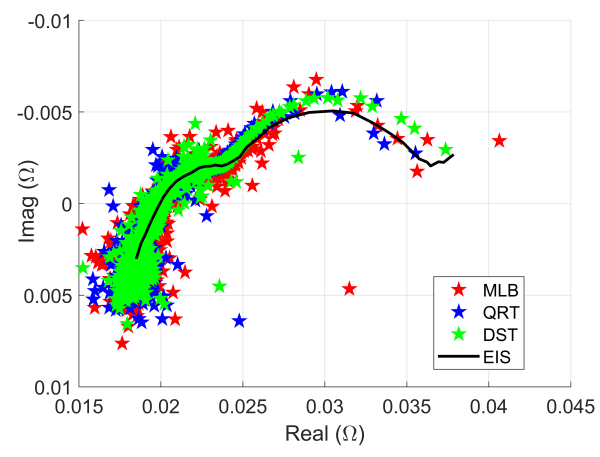

(c) SOC $60 \%$

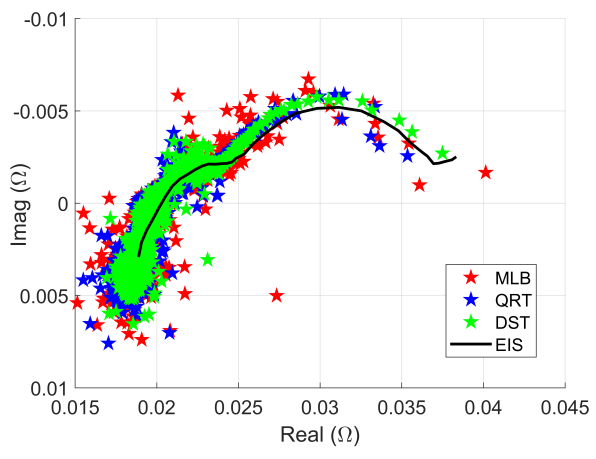

(b) SOC $40 \%$

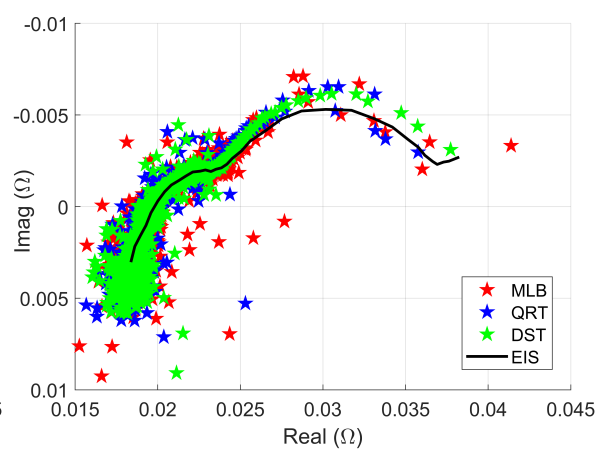

(d) SOC $80 \%$

Figure 4. Full-band impedance measurements in the complex-plane at different SOCs.

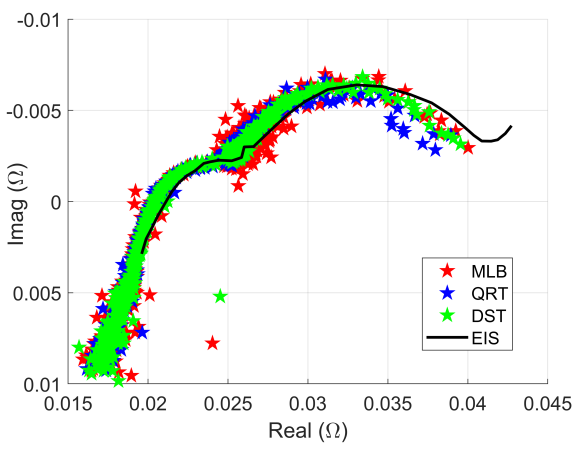

(a) SOC $20 \%$

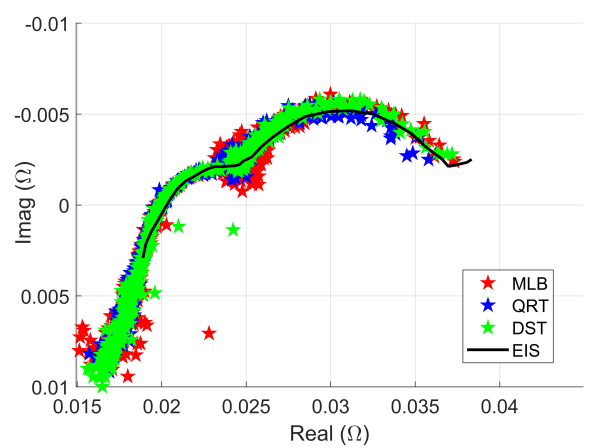

(c) SOC $60 \%$

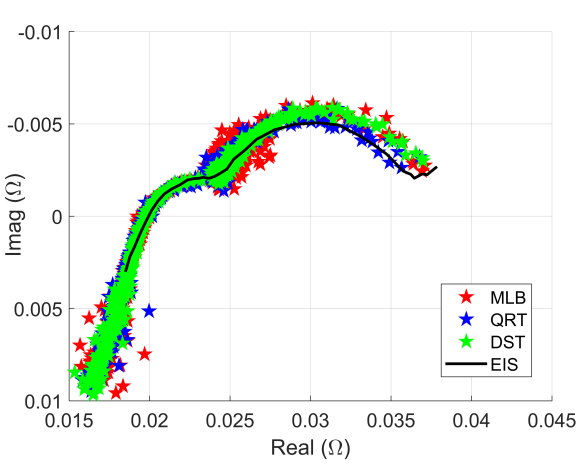

(b) SOC $40 \%$

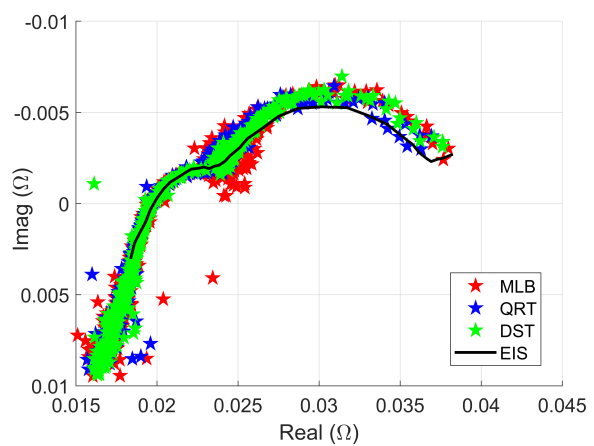

(d) SOC $80 \%$

Figure 5. Sub-band impedance measurements in the complex-plane at different SOCs. 


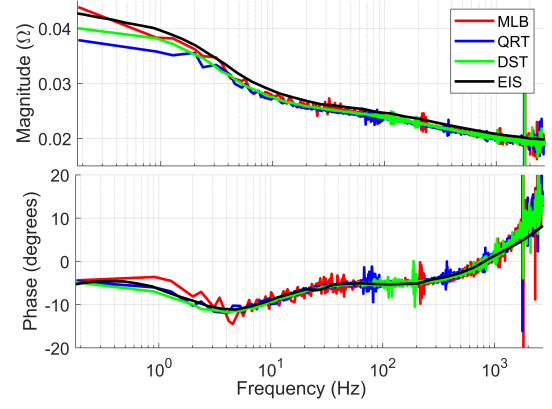

(a) Full-band SOC 20\%

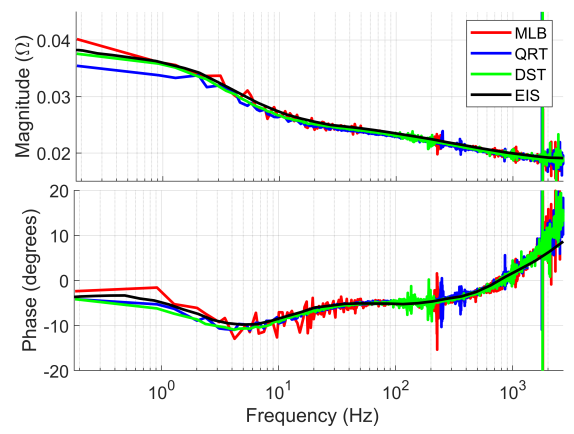

(c) Full-band SOC $40 \%$

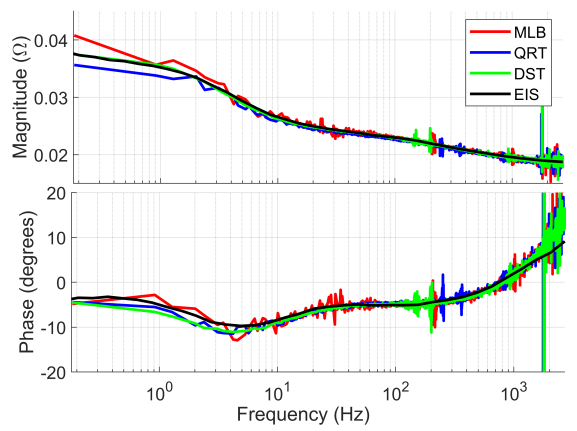

(e) Full-band SOC 60\%

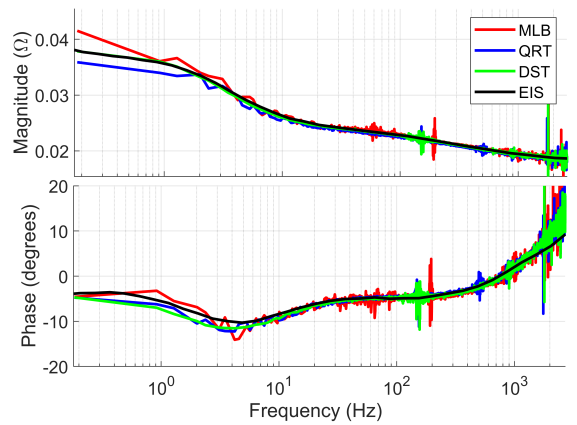

(g) Full-band SOC 80\%

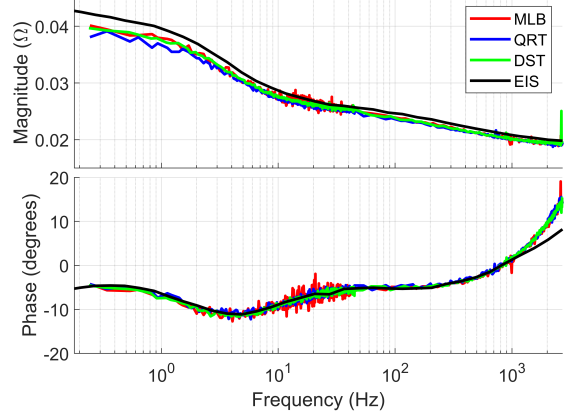

(b) Sub-band SOC 20\%

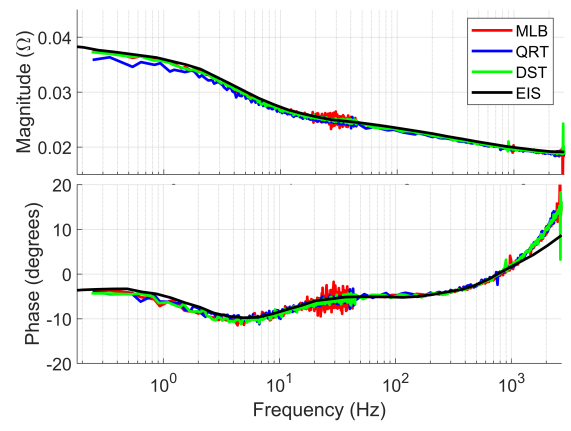

(d) Sub-band SOC $40 \%$

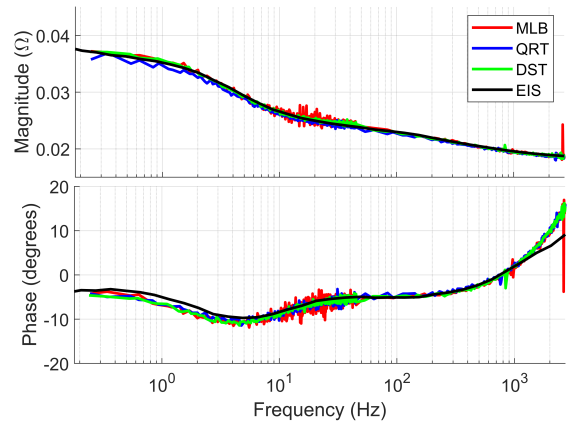

(f) Sub-band SOC 60\%

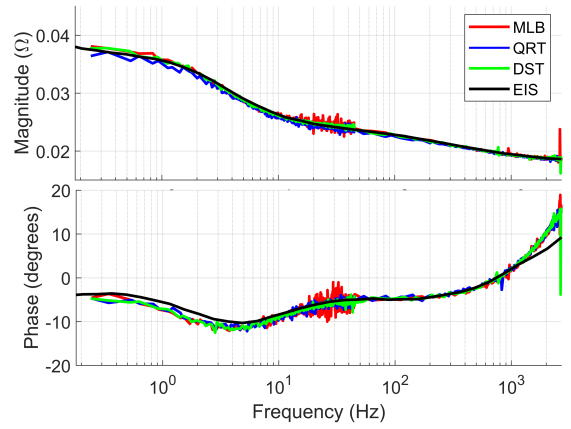

(h) Sub-band SOC $80 \%$

Figure 6. Bode plots of the full-band and sub-band impedance measurements at different SOCs.

The NRMSE values from the PRS measurement are presented in Table 2. The NRMSE values are the smallest for the DST measurements, which indicate the increased performance of the method compared to other PRSs. Moreover, the performance of all PRS sequences are increased when using sub-band measurements. The values at $20 \%$ of SOC are higher than for other SOCs, which is due to the fact that the measurement conditions are not linear anymore either for the PRSs or the EIS. 
Nevertheless, the NRMSE values indicate that the increased SNR of the sub-band measurements can increase the accuracy of the measurements and decrease the effect of nonlinear distortion for PRS measurements.

Table 2. NRMSE values of the PRS measurements (EIS as reference).

\begin{tabular}{|c|c|c|c|c|}
\hline & SOC $80 \%$ & SOC $60 \%$ & SOC $40 \%$ & SOC $20 \%$ \\
\hline \multicolumn{5}{|c|}{ Full-Band Measurements } \\
\hline MLB & $4.0 \%$ & $4.2 \%$ & $3.0 \%$ & $4.0 \%$ \\
\hline QRT & $4.5 \%$ & $4.2 \%$ & $5.7 \%$ & $8.1 \%$ \\
\hline DST & $1.7 \%$ & $1.5 \%$ & $2.0 \%$ & $5.0 \%$ \\
\hline \multicolumn{5}{|c|}{ Sub-Band Measurements } \\
\hline MLBS & $3.0 \%$ & $3.4 \%$ & $3.4 \%$ & $6.8 \%$ \\
\hline QRT & $3.8 \%$ & $4.0 \%$ & $5.9 \%$ & $8.8 \%$ \\
\hline$\tilde{\mathrm{DST}}$ & $1.4 \%$ & $1.5 \%$ & $1.8 \%$ & $5.7 \%$ \\
\hline
\end{tabular}

The validity of the PRS measurements is also analyzed in terms of the Kronig-Kramers compliance test. For the $\mathrm{K}-\mathrm{K}$ test, the data from the PRS measurements were reduced to cover the same amount of data points (33) as the EIS measurements. The harmonics from the PRS measurements that were the closest to the corresponding EIS harmonics were selected. The $\mathrm{K}-\mathrm{K}$ test results at $20 \%$ and $60 \%$ of SOC are shown in Figure 7 from full-band and sub-band measurements. For the full-band measurements, the fits are mostly having errors at the semicircle excluding the DST, which is accurately fitted to the corresponding data points from the measurements. For the the sub-band measurements, the fits are more coherently fitted throughout the bandwidth, which indicates the increased linearity and uniqueness of the measured PRS impedances compared to full-band measurements where only DST showed good performance. The EIS fits seem to have systematic error in the semicircle which indicates that the EIS data can be slightly corrupted.

The $\mathrm{K}-\mathrm{K}$ test residuals are shown at $20 \%$ and $60 \%$ of SOC in Figure 8 and the absolute mean residual values are shown in Table 3 at $20-80 \%$ of SOC for all perturbations. The residual plots of the DST method shows only single outlier points and no systematic error, which indicates good $\mathrm{K}-\mathrm{K}$ behavior. Apart from the DST, systematic error can be observed especially for the imaginary part residual plots for all other perturbation signals. Surprisingly, this can also be concluded for the EIS residuals and the systematic error of the EIS imaginary part residuals are observed to be significant. This is also concluded from Table 3, which indicates higher residual values than the DST measurements. For sub-band measurements, the mean residuals are slightly decreased from full-band measurements for all PRS methods. Despite the increased NRMSE values at $20 \%$ of SOC for all PRS, the mean residual values and the white-noise behavior of the residuals in Figure 8 indicate that the DST results are still more linear than the EIS. In total, the residuals are the lowest for the DST for both full-band and sub-band measurements, which also indicates the good performance of the DST signal. 
Table 3. K-K compliance test mean residuals

\begin{tabular}{|c|c|c|c|c|c|c|c|c|}
\hline \multicolumn{5}{|c|}{ Full-Band Sequence Measurements } & \multicolumn{4}{|c|}{ Sub-Band Sequence Measurements } \\
\hline \multicolumn{9}{|c|}{ SOC $20 \%$} \\
\hline & MLBS & QRT & DST & EIS & & MLBS & QRT & DST \\
\hline$\Delta_{\mathrm{re}}$ & $1.2 \%$ & $0.8 \%$ & $0.3 \%$ & $0.6 \%$ & $\Delta_{\mathrm{re}}$ & $1.0 \%$ & $0.7 \%$ & $0.5 \%$ \\
\hline$\Delta_{\mathrm{im}}$ & $1.2 \%$ & $0.8 \%$ & $0.4 \%$ & $0.8 \%$ & $\Delta_{\mathrm{im}}$ & $1.0 \%$ & $0.8 \%$ & $0.5 \%$ \\
\hline \multicolumn{9}{|c|}{ SOC $40 \%$} \\
\hline & MLBS & QRT & DST & EIS & & MLBS & QRT & DST \\
\hline$\Delta_{\mathrm{re}}$ & $1.0 \%$ & $0.7 \%$ & $0.4 \%$ & $0.6 \%$ & $\Delta_{\mathrm{re}}$ & $0.8 \%$ & $0.9 \%$ & $0.4 \%$ \\
\hline$\Delta_{\mathrm{im}}$ & $1.5 \%$ & $0.8 \%$ & $0.3 \%$ & $0.9 \%$ & $\Delta_{\mathrm{im}}$ & $0.9 \%$ & $0.9 \%$ & $0.5 \%$ \\
\hline \multicolumn{9}{|c|}{ SOC $60 \%$} \\
\hline & MLBS & QRT & DST & EIS & & MLBS & QRT & DST \\
\hline$\Delta_{\mathrm{re}}$ & $1.5 \%$ & $0.9 \%$ & $0.5 \%$ & $0.6 \%$ & $\Delta_{\mathrm{re}}$ & $0.9 \%$ & $0.8 \%$ & $0.4 \%$ \\
\hline$\Delta_{\mathrm{im}}$ & $1.3 \%$ & $0.7 \%$ & $0.3 \%$ & $0.9 \%$ & $\Delta_{\mathrm{im}}$ & $0.9 \%$ & $0.7 \%$ & $0.5 \%$ \\
\hline \multicolumn{9}{|c|}{ SOC $80 \%$} \\
\hline & MLBS & QRT & DST & EIS & & MLBS & QRT & DST \\
\hline$\Delta_{\mathrm{re}}$ & $1.3 \%$ & $1.1 \%$ & $0.5 \%$ & $0.6 \%$ & $\Delta_{\mathrm{re}}$ & $1.1 \%$ & $0.8 \%$ & $0.5 \%$ \\
\hline$\Delta_{\mathrm{im}}$ & $1.6 \%$ & $1.2 \%$ & $0.5 \%$ & $0.9 \%$ & $\Delta_{\mathrm{im}}$ & $0.9 \%$ & $0.7 \%$ & $0.4 \%$ \\
\hline
\end{tabular}

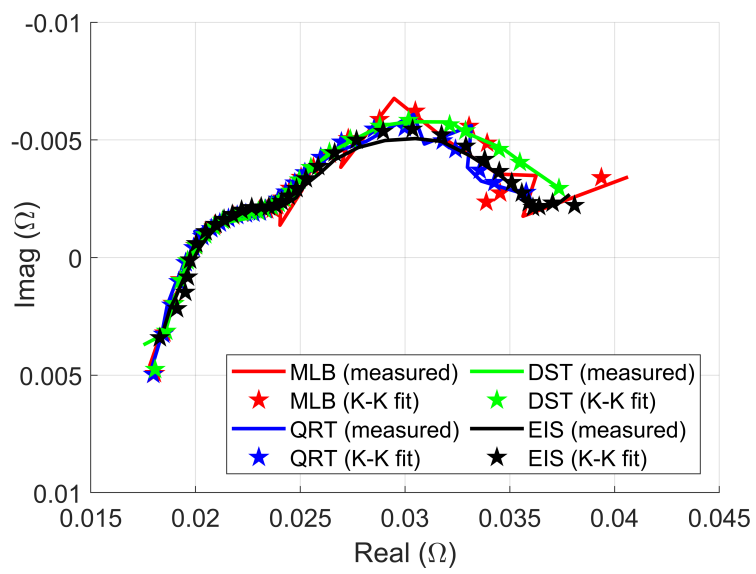

(a) Full-band SOC 60\%

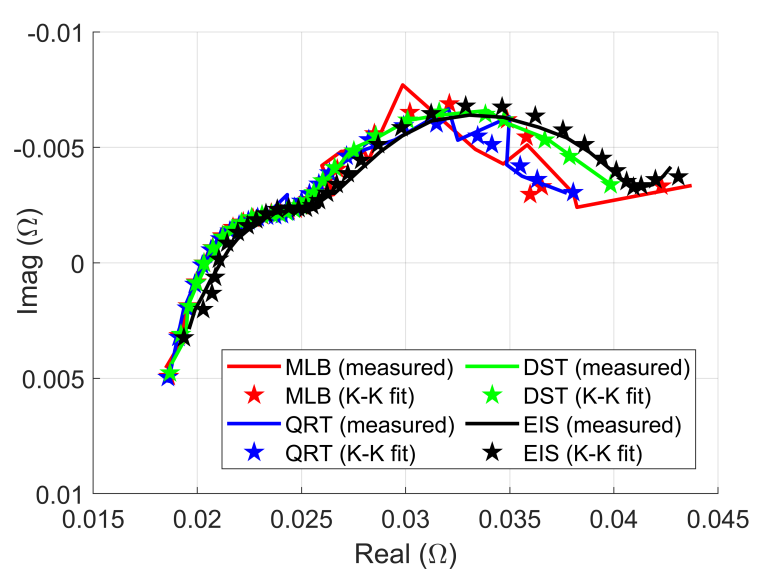

(c) Full-band SOC 20\%

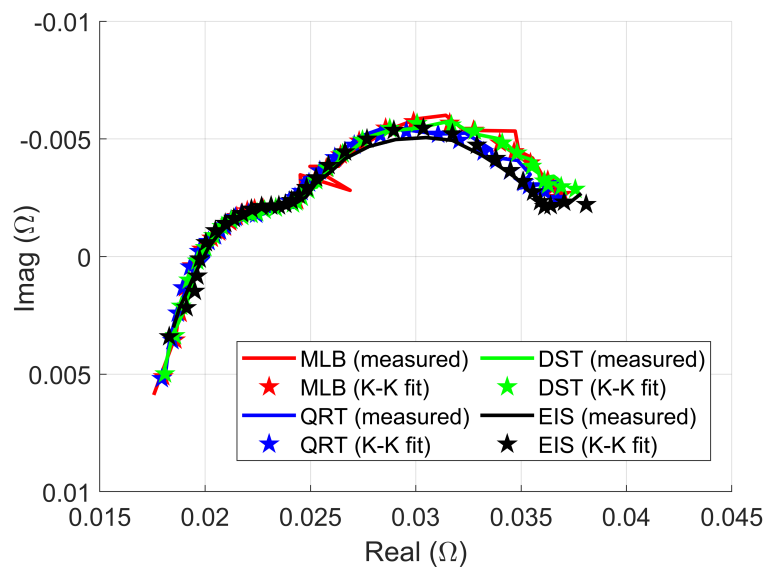

(b) Sub-band SOC 60\%

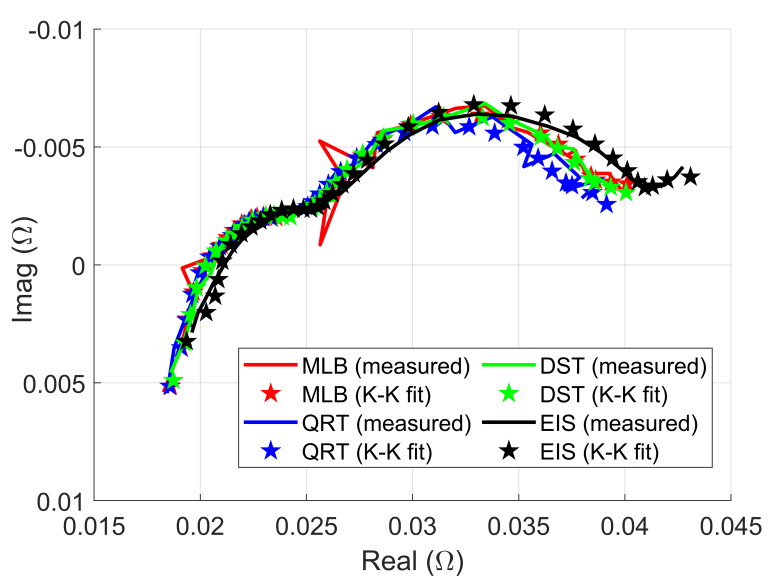

(d) Sub-band SOC $20 \%$

Figure 7. Measured and K-K fitted impedances from full-band and sub-band measurements at $20 \%$ and $60 \%$ of SOC. 


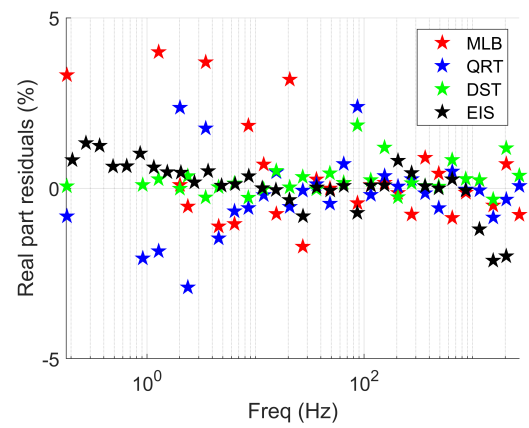

(a) Full-band $20 \%$ of SOC

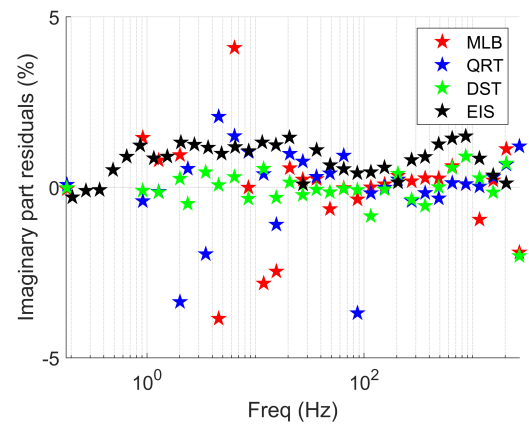

(c) Full-band $20 \%$ of SOC

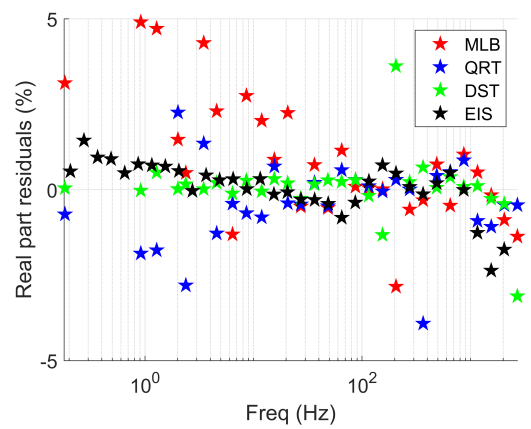

(e) Full-band $60 \%$ of SOC

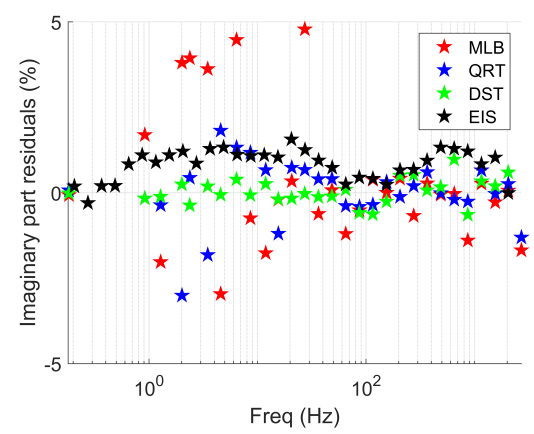

(g) Full-band $60 \%$ of SOC

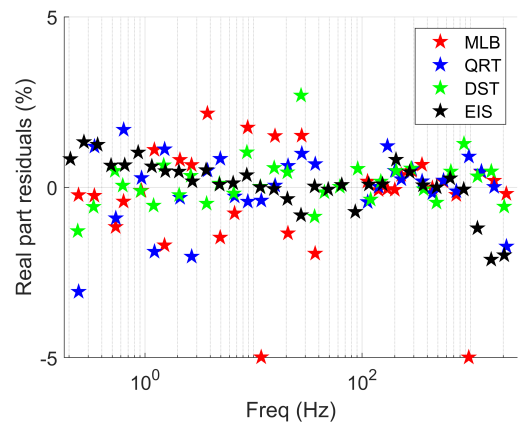

(b) Sub-band $20 \%$ of SOC

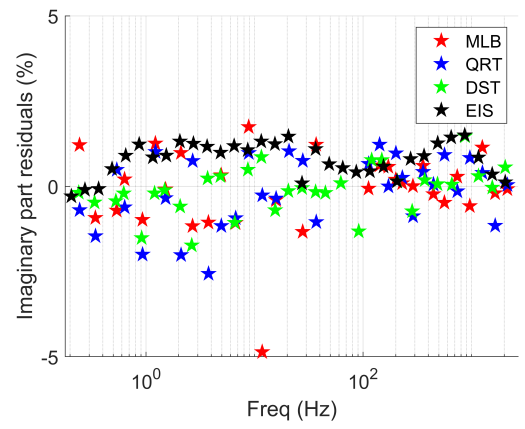

(d) Sub-band $20 \%$ of SOC

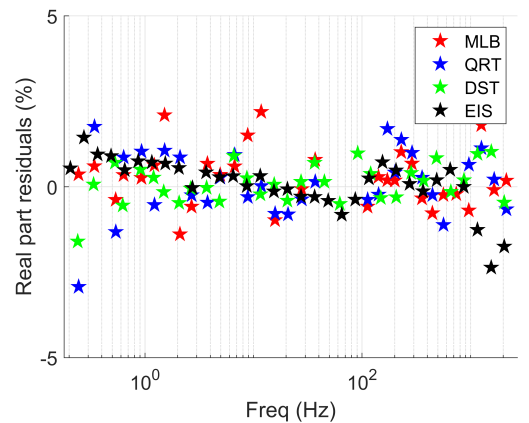

(f) Sub-band $60 \%$ of SOC

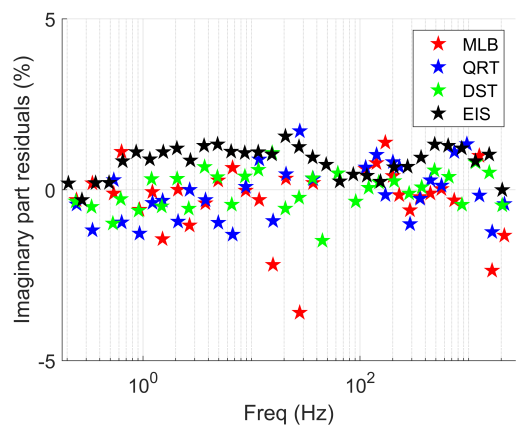

(h) Sub-band $60 \%$ of SOC

Figure 8. K-K compliance test residuals from impedance measurements at $20 \%$ and $60 \%$ of SOC.

To summarize the observations from the experiment results, the DST signal, with harmonic multiples two and three suppressed, increases the accuracy of the battery impedance measurements. This can be concluded both by the graphical analysis and the NRMSE values, which indicate that the DST sequence has the best performance compared to the two other pseudo-random sequences 
applied in this work. The Kronig-Kramers compliance test also indicates the good performance of the DST. Therefore, the DST impedance measurements have the potential to be used in the equivalent circuit models which can be further used for battery SOC and SOH estimation. In addition, the performance of the PRS methods was observed to increase when using sub-band measurements.

The K-K test results indicate that the EIS measurements may be corrupted. This can be caused by the long measurement time and the high current amplitude used for the measurements, which would imply that the measured EIS does not meet the linearity condition. Moreover, the used setup might be insufficient for the EIS measurements. Therefore, the traditional single-sine wave EIS measurements carried out in this paper might not be reliable. However, this is not affecting the validity of the DST method as indicated by the K-K test and the properties of the DST method discussed earlier. The corrupted results of the EIS can also indicate that the PRSs can be applied with slightly higher amplitude to still provide good impedances.

The measurement time of all PRS methods is approximately six seconds, while the measurement time for the EIS was $17 \mathrm{~min}$. Therefore, it can be stated that the PRS measurements are a lot faster than the EIS, although the measurement time of the EIS is increased by the heavy averaging (40 periods). However, even with the same amount of averaging than what was used for the PRS, the EIS measurement time with the used frequency harmonics would be $30 \mathrm{~s}$, which is still 5 times greater than the PRS measurement time.

\section{Conclusions}

Impedance measurement has become a popular method to characterize the dynamics of a Li-ion battery. In this paper, the use of the DST broadband sequence was demonstrated for the impedance measurements of an NMC battery cell. The DST sequence has harmonic multiples of two and three suppressed, which results in an increased performance under system nonlinear effects. The performance of the method was compared to the conventional EIS method, as well as to other PRS signals, the MLB, and the QRT. The results showed that the DST provides the lowest measurement error to the EIS. Moreover, the DST method was found to be the most compliant to the Kronig-Kramers impedance validation test. In addition, it was shown that the use of adjacently measured sub-bands can increase the accuracy of the PRS measurements and also reduce the effect of nonlinear distortion dynamics for the MLB and QRT measurements. Due to the good performance under battery nonlinearities, low measurement time and simplicity, the DST method is well suited to be used as a perturbation signal for impedance measurements in practical battery applications.

Author Contributions: Conceptualization, J.S.; methodology, J.S. and T.R.; data curation, J.S. and D.-I.S.; writing-original draft preparation, J.S.; writing—review and editing, T.R. and D.-I.S.; supervision, T.R. and D.-I.S. All authors have read and agreed to the published version of the manuscript.

Funding: This research received no external funding.

Conflicts of Interest: The authors declare no conflict of interest. The authors are responsible for the content and writing of this article.

\section{Abbreviations}

The following abbreviations are used in this manuscript:

$\begin{array}{ll}\text { DST } & \text { Direct synthesis ternary } \\ \text { EIS } & \text { Electrochemical impedance spectroscopy } \\ \text { MLB } & \text { Maximum length binary } \\ \text { NMC } & \text { Nickel manganese cobalt } \\ \text { NRMSE } & \text { Normalized root mean square error } \\ \text { PRS } & \text { Pseudo random sequence } \\ \text { QRT } & \text { Quadratic residue ternary } \\ \text { SNR } & \text { Signal-to-noise ratio } \\ \text { SOC } & \text { State of charge } \\ \text { SOH } & \text { State of health }\end{array}$




\section{References}

1. Chemali, E.; Preindl, M.; Malysz, P.; Emadi, A. Electrochemical and Electrostatic Energy Storage and Management Systems for Electric Drive Vehicles: State-of-the-Art Review and Future Trends. IEEE J. Emerg. Sel. Top. Power Electron. 2016, 4, 1117-1134. [CrossRef]

2. Chen, Z.; Fu, Y.; Mi, C.C. State of Charge Estimation of Lithium-Ion Batteries in Electric Drive Vehicles Using Extended Kalman Filtering. IEEE Trans. Veh. Technol. 2013, 62, 1020-1030. [CrossRef]

3. Berecibar, M.; Gandiaga, I.; Villarreal, I.; Omar, N.; Van Mierlo, J.; Van den Bossche, P. Critical review of state of health estimation methods of Li-ion batteries for real applications. Renew. Sustain. Energy Rev. 2016, 56, 572-587. [CrossRef]

4. Westerhoff, U.; Kroker, T.; Kurbach, K.; Kurrat, M. Electrochemical impedance spectroscopy based estimation of the state of charge of lithium-ion batteries. J. Energy Storage 2016, 8, 244-256. [CrossRef]

5. Lasia, A. Electrochemical Impedance Spectroscopy and Its Applications; Springer: New York, NY, USA, $2014 ;$ p. 369.

6. Barai, A.; Chouchelamane, G.H.; Guo, Y.; McGordon, A.; Jennings, P. A study on the impact of lithium-ion cell relaxation on electrochemical impedance spectroscopy. J. Power Sources 2015, 280, 74-80. [CrossRef]

7. Karden, E.; Buller, S.; De Doncker, R.W. A method for measurement and interpretation of impedance spectra for industrial batteries. J. Power Sources 2000, 85, 72-78. [CrossRef]

8. Howey, D.A.; Mitcheson, P.D.; Member, S.; Yufit, V.; Offer, G.J.; Brandon, N.P. Online Measurement of Battery Impedance Using Motor Controller Excitation. IEEE Trans. Veh. Technol. 2014, 63, 2557-2566. [CrossRef]

9. Al Nazer, R.; Cattin, V.; Granjon, P.; Montaru, M.; Ranieri, M. Broadband identification of battery electrical impedance for HEVs. IEEE Trans. Veh. Technol. 2013, 62, 2896-2905. [CrossRef]

10. Sihvo, J.; Stroe, D.; Messo, T.; Roinila, T. Fast Approach for Battery Impedance Identification Using Pseudo-Random Sequence Signals. IEEE Trans. Power Electron. 2020, 35, 2548-2557. [CrossRef]

11. Fairweather, A.J.; Foster, M.P.; Stone, D.A. Battery parameter identification with Pseudo Random Binary Sequence excitation (PRBS). J. Power Sources 2011, 196, 9398-9406. [CrossRef]

12. Barsoukov, E.; Macdonald, J.R. (Eds.) Impedance Spectroscopy: Theory, Experiment, and Applications, 2nd ed.; John Wiley \& Sons, Inc.: Hoboken, NJ, USA, 2005.

13. Godfrey, K. Perturbation Signals for System Identification; Prentice Hall: Upper Saddle River, NJ, USA, 1993.

14. Godfrey, K.; Barker, H.; Tucker, A. Comparison of perturbation signals for linear system identification in the frequency domain. IEE Proc. Control Theory Appl. 1999, 146, 535-548. [CrossRef]

15. Tan, A.H.; Godfrey, K.R. The generation of binary and near-binary pseudorandom signals: An overview. IEEE Trans. Instrum. Meas. 2002, 51, 583-588. [CrossRef]

16. Godfrey, K.; Barker, H.; Tan, A. Ternary input signal design for system identification. IET Control Theory Appl. 2007, 1, 1224-1233.

17. Tan, A.H. Direct synthesis of pseudo-random ternary perturbation signals with harmonic multiples of two and three suppressed. Automatica 2013, 49, 2975-2981. [CrossRef]

18. Roinila, T.; Vilkko, M.; Suntio, T. Frequency-response measurement of switched-mode power supplies in the presence of nonlinear distortions. IEEE Trans. Power Electron. 2010, 25, 2179-2187. [CrossRef]

19. Schönleber, M.; Klotz, D.; Ivers-Tiffée, E. A Method for Improving the Robustness of linear Kramers-Kronig Validity Tests. Electrochim. Acta 2014, 131, 20-27. [CrossRef]

20. Boukamp, B.A. A Linear Kronig-Kramers Transform Test for Immittance Data Validation. J. Electrochem. Soc. 1995, 142, 1885-1894. [CrossRef]

21. Giner-Sanz, J.J.; Ortega, E.M.; Pérez-Herranz, V. Optimization of the Perturbation Amplitude for EIS Measurements Using a Total Harmonic Distortion Based Method. J. Electrochem. Soc. 2018, 165, E488-E497. [CrossRef]

(C) 2020 by the authors. Licensee MDPI, Basel, Switzerland. This article is an open access article distributed under the terms and conditions of the Creative Commons Attribution (CC BY) license (http:/ / creativecommons.org/licenses/by/4.0/). 